\title{
Consideraciones bioéticas del tamizaje neonatal: pautas para su regulación integral ${ }^{*}$
}

\author{
Bioethical issues of neonatal screening: \\ guidelines for its comprehensive regulation
}

\section{As considerações bioéticas da triagem neonatal: orientações para a seu regulamentação integral}

\author{
Fecha de recepción: 15 de junio de 2015 \\ Fecha de evaluación: 18 de agosto de 2015 \\ Fecha de aceptación: 26 de octubre de 2015 \\ Disponible en línea: 15 de diciembre de 2015
}

\author{
Ricardo A. Cifuentes*
}

DOI: http://dx.doi.org/10.18359/rlbi.1445

\section{Cómo citar:}

Cifuentes, R. A. (2016). Consideraciones bioéticas del tamizaje neonatal: pautas para su regulación integral. Revista Latinoamericana de Bioética, 16(1), 154-173. DOI: http://dx.doi.org/10.18359/rlbi.1445

Artículo de Revisión. Trabajo realizado durante la descarga concedida por la Universidad Militar Nueva Granada para adelantar el Doctorado en Bioética en esta institución.

** Médico cirujano, especialista en Gerencia de la Salud Pública, M.Sc. en Genética Humana, M.Sc. en Informática Médica; estudiante del Doctorado en Bioética, de la Universidad Militar Nueva Granada, Bogotá, Colombia. Profesor asociado, de la Universidad Militar Nueva Granada. Correo electrónico: ricardo.cifuentes@unimilitar.edu.co 


\section{Resumen}

El tamizaje neonatal pretende detectar al nacimiento anomalías susceptibles de tratamiento antes de la aparición de enfermedad. Su objetivo es evitar discapacidad e incluso la muerte de algunos recién nacidos. .Con los desarrollos tecnológicos los beneficios se han extendido a la familia y a la sociedad. Contrariamente, también se han documentado daños psicológicos, sociales e incluso físicos. La probabilidad de daño está influenciada por consideraciones asociadas con principios bioéticos como la confidencialidad, la cual se relaciona con los principios de dignidad y autonomía. Aparecen entonces controversias como el tiempo de retención de muestras. De forma similar, hay otras controversias asociadas a consideraciones bioéticas como la prioridad de la persona o el consentimiento. A pesar del panorama descrito, la legislación no aborda de forma integral las consideraciones bioéticas. Colombia no es la excepción; la normatividad específica para tamizaje neonatal no considera ni siquiera de forma aislada consideraciones fundamentales en este tópico. En este contexto, el derecho blando da directivas como el deber de garantizar el acceso o las circunstancias en las cuales es posible realizar el tamizaje neonatal sin beneficio directo para el neonato. Sin embargo, no aborda otras consideraciones como el consentimiento y la confidencialidad, lo que amerita un debate bioético integral.

Palabras clave: tamizaje neonatal, bioética, pruebas genéticas, legislación como asunto.

\section{Abstract}

The neonatal screening aims to detect anomalies at birth that are susceptible to treatment before the onset of disease. Its goal: to prevent disability and even death of some newborns. With technological developments, the benefits have extended to the family and society. Conversely, it has also been documented damages such as psychological, social and even physical harms. The probability of damage is influenced by issues associated with bioethical principles such as confidentiality, which is related to the principles of Dignity and Autonomy. Controversies such as the retention time of samples then appear. Similarly, there are other controversies associated with bioethical issues such as the priority of the person or consent. Despite the described panorama, the legislation does not address the bioethical issues comprehensively. Colombia is not an exception; specific regulation for neonatal screening does not consider, even in isolation, fundamental issues in this topic. In this context, the soft-law gives directives as the duty to ensure access to or the circumstances in which it is possible to perform neonatal screening without direct benefit to the neonate. However, it does not address other issues such as consent and confidentiality thus deserving a comprehensive bioethical debate.

Keywords: Neonatal Screening, Bioethics, Genetic Testing, Legislation as a topic

\section{Resumo}

A triagem neonatal visa detectar ao nascimento anomalias suscetíveis de tratamento antes do aparecimento da doença. O seu objetivo é prevenir a incapacidade e até mesmo a morte de alguns recém-nascidos. Com os desenvolvimentos tecnológicos os benefícios para a família foram estendidos e também para a sociedade. Contrariamente, também têm sido documentados danos psicológicos, sociais e até mesmo danos físicos. A probabilidade de dano é influenciada por considerações associadas com princípios bioéticos, tais como a confidencialidade, que se relaciona com os princípios da dignidade e autonomia. Aparecem então controvérsias, como o tempo de retenção de amostras. Da mesma forma, há outras controvérsias associadas a considerações bioéticas como a prioridade da pessoa ou do consentimento. Apesar da situação descrita, a legislação não aborda de forma abrangente as considerações bioéticas. Colômbia não é uma exceção; a normatividade específica para a triagem neonatal não considera nem sequer de forma isolada considerações fundamentais sobre este tema. É neste contexto, que o direito macio dá diretivas como o dever de garantir o acesso ou as circunstâncias nas quais é possível realizar a triagem neonatal sem nenhum beneficio direto para o recém-nascido. No entanto, ele não aborda outras considerações, como o consentimento e a confidencialidade, o que o leva a merecer um debate bioético abrangente.

Palavras-chave: triagem neonatal, bioética, probas genéticas, legislação como assunto. 


\section{Introducción}

La medicina pretende pasar de un enfoque dirigido a diagnosticar y tratar a uno basado en predecir y prevenir las enfermedades (Loscalzo, Kohane y Barabasi, 2007). En este ámbito se destaca el tamizaje neonatal, actividad que consiste en detectar anomalías en los recién nacidos de una población antes de que se hagan evidentes como enfermedades y la cual es realizada masivamente en países como Estados Unidos, donde cubre anualmente alrededor de cuatro millones de individuos (Rose y Dolan, 2012).

El tamizaje neonatal se realiza desde la década de los sesenta del siglo XX cuando se desarrolló una prueba de laboratorio para la detección precoz de la fenilcetonuria (Ortiz, 2008). Nació entonces esta actividad basándose en la detección de metabolitos propios de anomalías susceptibles de manejo temprano (Schulman, 2008). Las anomalías por detectar corresponden generalmente a alteraciones genéticas como la fenilcetonuria, enfermedad caracterizada por toxicidad neurológica debida a la acumulación de fenilalanina y sus metabolitos como el fenilpiruvato. Consecuencias como el retardo mental pueden evitarse con una dieta deficitaria en fenilalanina desde el nacimiento (Rose y Dolan, 2012).

Recientemente el tamizaje neonatal ha mostrado un cambio de foco: ahora su objeto no son solo anomalías cuyo tratamiento desde el nacimiento previene discapacidad o muerte en el paciente (President's Council on Bioethics, 2008). En los primeros años del siglo XXI el Colegio Americano de Genética Médica (ACMG, por sus iniciales en inglés) recomendó incluir también en el tamizaje neonatal pruebas que demuestren beneficios de una detección temprana para la familia o la sociedad, como el descubrimiento de portadores y planeación de la familia. Consecuentemente, la recomendación en Estados Unidos es tamizar al nacimiento 29 enfermedades centrales y 25 enfermedades secundarias (Schulman, 2008). Esta recomendación se ha seguido gracias a la técnica de espectrometría de masas en tándem (MS/MS), capaz de detectar múltiples metabolitos en una sola muestra de sangre (Campos, 2010).

No obstante la reciente ampliación, parece inevitable una mayor expansión del panel de enfermedades por tamizar al nacimiento, debido a iniciativas como la del Instituto $\mathrm{Na}$ cional de Salud Infantil y Desarrollo Humano de los Estados Unidos. Esta entidad considera pasar de métodos fenotípicos como la espectrometría a plataformas basadas en estudios genómicos (Schulman, 2008). Así, mediante técnicas de secuenciamiento de nueva generación se puede analizar la totalidad del genoma, del exoma (parte codificante de todos los genes) o un panel de genes a la medida de lo requerido (Raffan y Semple, 2011). Se podrían entonces detectar desde el 
nacimiento anomalías sin tratamiento o que se manifestarán más tarde en la vida, o identificar personas que simplemente tienen un riesgo aumentado de presentar enfermedades comunes (Orzalesi y Danhaive, 2009).

\section{Daños relacionados con el tamizaje neonatal}

En el contexto de una ampliación de beneficios se debe estar consciente de los daños que se presentan en el tamizaje neonatal. Se pueden dar diferentes tipos de daños como consecuencia de un resultado positivo, es decir, una prueba que señala la presencia de una enfermedad o el estado de portador de una mutación que predispone a enfermedad. Esto aplica tanto en el caso de ser un resultado falso positivo como incluso un verdadero positivo (ver figura 1). Cuando no existe una justificación para este daño se estaría vulnerando el principio de no-maleficencia, como podría darse en algunos casos en los que se puede hacer el diagnóstico al nacimiento de una anomalía para la cual no haya posibilidad de tratamiento o este sea de eficacia discutible.

Figura 1. Diagrama de daños relacionados con el tamizaje neonatal.

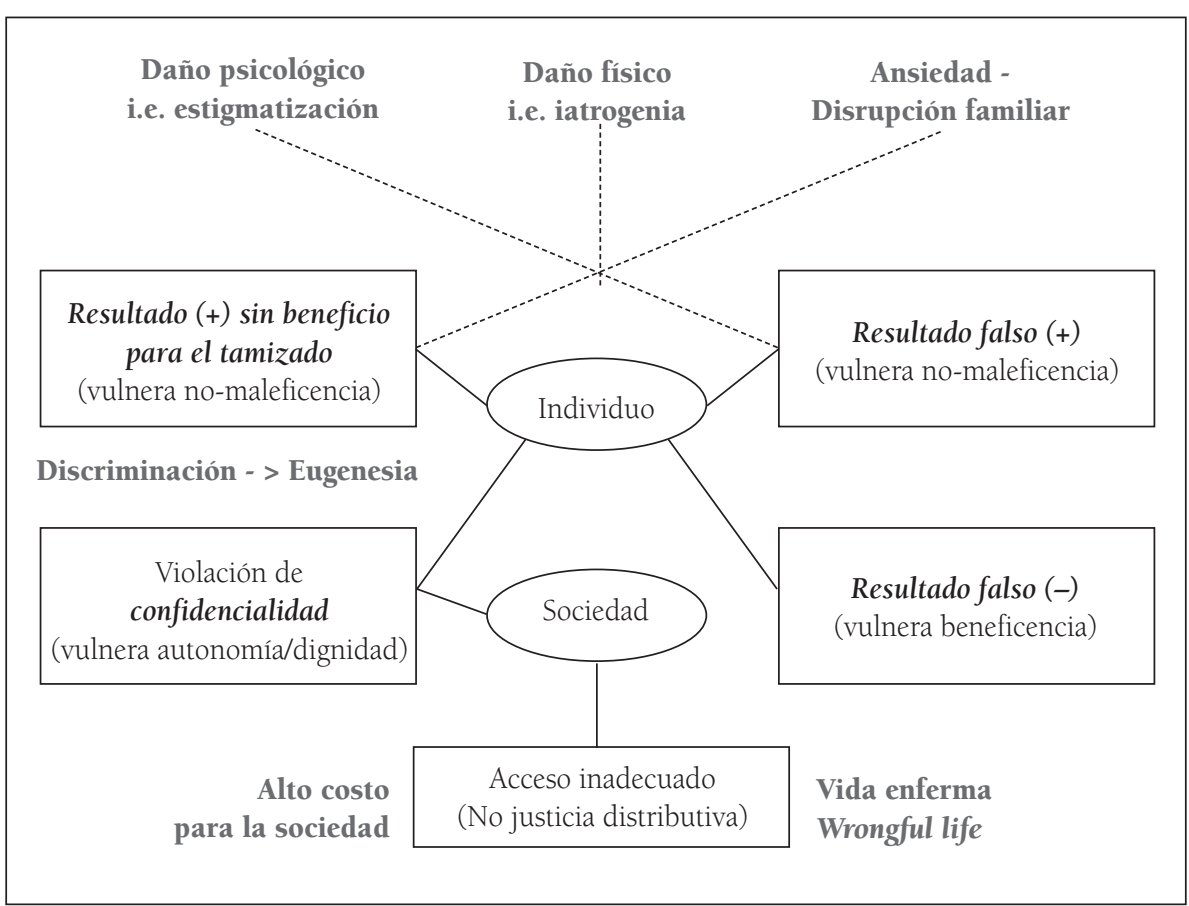

Fuente: elaboración propia. 
Hay enfermedades como la de Krabbe, de difícil diagnóstico y cuyo único tratamiento, el trasplante de células madre hematopoyéticas, no siempre es efectivo, pero sí conlleva un riesgo vital importante (Lantos, 2011). Otro tipo de daño físico es el que puede darse como consecuencia del aumento de probabilidad de una enfermedad. Hay reportes como el de una menor de 13 años portadora de una mutación en TP53 que implica aumento del riesgo de cáncer. Sin importar que en una ocasión haya presentado un cuadro clínico compatible con un quiste de características no agresivas, pediatras oncólogos estuvieron de acuerdo por su antecedente en realizar una biopsia ósea con reconstrucción mediante injerto para evaluar una lesión que resultó benigna (Schiffman et al., 2013).

Pueden presentarse también otros tipos de daños. Se han descrito afectaciones psicológicas como la ansiedad generada al ser portador de mutaciones que incrementan el riesgo de presentar un tipo de cáncer a pesar de ser incierta la aparición de esta enfermedad. Otro tipo de daño es el social o familiar, como el que se puede dar cuando una prueba indica que un neonato es portador de una mutación que predispone a una enfermedad. Esta información es de beneficio para la familia al permitir a sus padres tomar decisiones reproductivas como evitar un nuevo embarazo o considerar el uso de reproducción asistida por un donante. Sin embargo, podría afectar la forma como es visto el menor en su familia o la forma como se relaciona con potenciales parejas (Almond, 2006).

Adicionalmente, la noción de beneficio para la familia también genera la preocupación respecto a hasta dónde puede el tamizaje neonatal afectar la dignidad humana. Si se llega a evitar por métodos como el aborto el nacimiento de hermanos afectados de un menor en quien se detectó una mutación patogénica, el tamizaje neonatal sería moralmente cuestionable. Se estaría promoviendo un comportamiento eugenésico no solo a nivel individual sino también social, y se eliminarían humanos con una determinada condición (Schulman, 2008).

Puede presentarse también una dudosa eugenesia, generalmente por los padres, quienes podrían tomar decisiones erróneas basadas en considerar equivocadamente como patogénicas mutaciones con un efecto clínico nulo o al menos incierto. Al respecto, es relevante el hallazgo de un número importante de mutaciones completamente funcionales en genes implicados en conocidas enfermedades (Raffan y Semple, 2011).

Otra preocupación del ámbito familiar es el impacto de resultados erróneos. En el caso de falsos-positivos en el tamizaje neonatal, a pesar de la corrección del error, se ha observado disfunción de relaciones padre-hijo y ansiedad de los padres por la salud de su hijo (Gurian et al., 2006), y se han documentadp el 
doble de hospitalizaciones en estos niños (Tarini, 2007). En el caso de resultados falsos negativos, la equivocación llevaría a no descubrir anomalías que pueden repercutir en enfermedades discapacitantes con la consiguiente afectación económica que acarrea una vida enferma, wrongful life (Emaldi-Cirión, 2002). Igual consecuencia podría tener la falta de acceso a una prueba de reconocida utilidad clínica.

\section{Consideraciones bioéticas en el tamizaje neonatal}

La probabilidad de ocurrencia de daños relacionados con el tamizaje neonatal no solo está influenciada por datos erróneos que amenazan su validez, sino también por otras consideraciones relacionadas con principios bioéticos que afectan la realización de esta actividad (ver figura 2).

Figura 2. Consideraciones bioéticas del tamizaje neonatal.

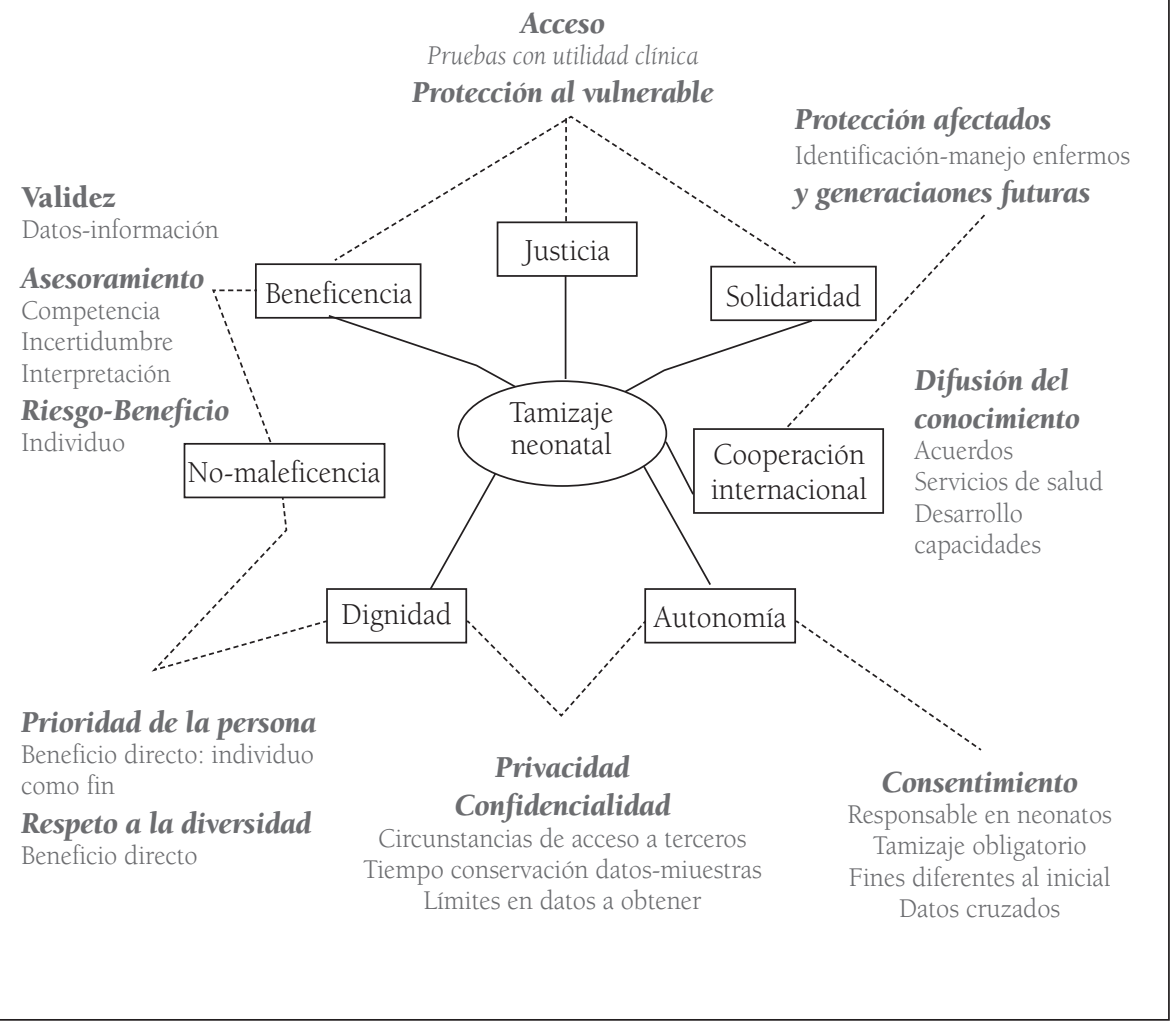

Fuente: elaboración propia. 
Una de estas consideraciones bioéticas es la prioridad de la persona lo que justifica efectuar el tamizaje cuando hay un beneficio directo para el neonato, por ejemplo la disponibilidad de tratamiento para la anomalía por detectar. En este caso, no realizar una prueba genético-molecular para establecer la presencia de una anomalía susceptible de tratamiento omite una acción de carácter obligatorio para prevenir la ocurrencia de un daño (Beauchamp y Childress, 2009a), vulnerando el principio de beneficencia.

Actualmente, hay consenso internacional respecto a la adecuada relación riesgo-beneficio de realizar tamizaje neonatal para detectar preclínicamente enfermedades con tratamiento disponible (Schulman, 2008). Son ejemplos de estas enfermedades la fenilcetonuria, la deficiencia de biotinidasa o la galactosemia, que pueden ser tratadas antes de la aparición de síntomas y evitar así daños severos en órganos como el cerebro o el hígado (Rose y Dolan, 2012).

En contraste, es controversial realizar tamizaje con beneficio para la familia o la sociedad, pero sin beneficio directo para el neonato, como puede ser realizarlo en enfermedades sin tratamiento disponible (Elliman, 2012). Así, un progreso científico para la sociedad como lo es la investigación de nuevos tratamientos podría justificar la realización del tamizaje (Schulman, 2008). No obstante, una investigación de esta naturaleza podría vulnerar la dignidad humana al instrumentalizar al hombre (Gómez, 2010). Incluso, de ocasionarse un daño no justificado como la discriminación se vulneraría también el principio de no-maleficencia.

Otra característica del tamizaje neonatal es que las enfermedades por predecir no son iguales. A grandes rasgos pueden clasificarse en dos grupos: monogénicas que en general se predicen al encontrar una mutación que se conoce que está relacionada con una patología específica y multifactoriales en las cuales la predicción no solo tiene en cuenta la carga genética, sino también otros elementos como la influencia ambiental (Strachan y Read, 2011). Se evidencia así otro aspecto del tamizaje neonatal: la incertidumbre en la predicción, la cual se considera menor en la predicción de enfermedades monogénicas que en las multifactoriales. Sin embargo, una característica genética como la penetrancia, porcentaje de la población con una mutación que presenta la enfermedad (Jonas et al., 2012), ilustra la diferente incertidumbre de predicción incluso entre enfermedades monogénicas.

Se vislumbra entonces otra consideración bioética: la necesidad de un adecuado asesoramiento que maneje la incertidumbre e interpretación de los resultados de pruebas. Como se ha descrito en fibrosis quística, algunas pruebas incluyendo el análisis de mutaciones no son concluyentes (Massie 
y Gillam, 2014). Incluso mutaciones con comprobada alteración funcional frecuentemente solo cambian la susceptibilidad a una enfermedad de forma variable (Schulman, 2008). Adicionalmente, con las nuevas tecnologías la interpretación de una cantidad masiva de datos genético-moleculares se hace más compleja (Raffan y Semple, 2011).

Desconocer la incertidumbre siendo determinista puede ocasionar un daño no justificado y vulnerar así el principio de no-maleficencia (Beauchamp y Childress, 2009b). Son ejemplos de lo anterior los falsos positivos que llevan a tratamientos innecesarios o afectan injustificadamente la relación entre padres e hijos. Por otro lado, a pesar de que el tamizaje busca optimizar la sensibilidad, pueden presentarse falsos negativos (Tarini, 2007). En este caso desconocer la incertidumbre para descartar la enfermedad favorecería daños por omitir un manejo necesario y de esta manera se transgrediría el principio de beneficencia.

Por otra parte, una prueba con implicaciones predictivas debe tener consentimiento del individuo al que se le realiza en aras de respetar dentro de su autonomía el "derecho a no saber", al cual se le ha dado una discutible posición prominente sobre otros intereses (Helgesson et al., 2007). No obstante, al corresponder los evaluados a neonatos que no tienen el control de la decisión respecto a ser tamizados, la Organización Mundial de la Salud (OMS) establece en sus guías que el tamizaje neonatal debe ser mandatorio y sin costo si el diagnóstico temprano y tratamiento benefician al recién nacido (Kerruish y Robertson, 2005). En este ámbito, hay gran preocupación respecto a la violación de la privacidad familiar y la pérdida de autonomía paterna (Hendrix et al., 2013).

El tamizaje obligatorio parece aceptable bajo algunas condiciones, pero la educación de los padres y la oportunidad para rechazar el tamizaje deben ser parte del sistema. El consentimiento informado es necesario si hay incertidumbre acerca de los beneficios de la prueba para el menor (Matsuda, 2003), pero no debe olvidarse que una buena comunicación con la familia puede mejorar la relación riesgo-beneficio no solo previa al consentimiento, sino luego del tamizaje, con lo cual se busca disminuir los daños psicosociales (Farrell et al., 2011).

Por otro lado, es indiscutible la necesidad del consentimiento de los padres para la realización del tamizaje de anomalías sin beneficio para el neonato. Dado que las nuevas tecnologías en genética permiten evaluar muchas predisposiciones y anomalías heterogéneas simultáneamente, es un reto proveer una información adecuada antes de una prueba y obtener una decisión autónoma (Bunnik et al., 2013). Además, deben analizarse las ventajas y desventajas de tamizar neonatos con base en el consentimiento de los padres respecto 
a enfoques alternativos, como hacer un tamizaje a los futuros padres para detectar el estado de portadores (De Wert, 2005), teniendo en cuenta que ellos sí podrían tomar la decisión con autonomía.

Una controversia interesante es el debate respecto a tamizar enfermedades de inicio en la edad adulta. La academia americana de pediatría considera que el tamizaje genético de estas enfermedades debe diferirse, mientras que el Colegio Americano de Genética Médica (ACMG, por sus siglas en inglés) recomienda la evaluación de los menores para mínimo algunas enfermedades de edad en la adultez; esto lo justifica en el hecho de que se busca el mejor interés para el menor y su familia (Hardart y Chung, 2014).

Igualmente, una vez obtenidos los resultados debe mantenerse su confidencialidad respetando el "derecho a la intimidad" (Sánchez, 2002). Al respecto, aparece la preocupación de hasta qué grado es posible mantener la confidencialidad. En primer lugar, hay un discutible pero aparente consenso en que un estado de portador descubierto incidentalmente en el neonato no puede ocultarse a los padres (Miller et al., 2009).

Por otra parte, está tomando especial relevancia el establecimiento de biobancos de muestras del neonato, como los de DNA y células de sangre de cordón umbilical típicamente previo consenti- miento informado de los padres (Chase et al., 1998). Padres encuestados no solo piden que las muestras se desliguen de la identidad de sus hijos, sino también que solicite su consentimiento para cada uso diferente de las muestras (Hendrix et al., 2013). La visión opuesta no ha generado tanta preocupación; sin embargo, no debe olvidarse que muestras almacenadas son un recurso público valioso que puede llevar a mejoras en salud pública (Lewis et al., 2012). Se destaca entonces la importancia de informar a los padres del valor potencial de investigación de las muestras y darles la seguridad de que los resultados de la investigación no serán ligados a ningún neonato en particular (Matsuda, 2003).

Otra consideración bioética es la posibilidad de acceso a pruebas genético-moleculares del tamizaje neonatal con validez y utilidad clínica. En este caso, la mayor preocupación ha sido asegurar la atención médica (Burke et al., 2001), es decir la protección de los afectados bajo el principio de solidaridad. Sin embargo, hay diferencias en el acceso; para citar un ejemplo, mientras algunos países tamizan pocas enfermedades otros lo hacen en el orden de docenas (Elliman, 2012), lo cual justifica su análisis en el contexto de la difusión del conocimiento, consideración relacionada con el principio bioético de cooperación internacional.

No obstante, son llamativas las diferencias de acceso en países de la misma región. En el caso de la fenilcetonu- 
ria, algunos países latinoamericanos realizan el tamizaje: Argentina, Brasil, Chile, Costa Rica, Cuba, Guatemala, Paraguay, Uruguay, Venezuela (Raho, 2008), mientras otros países de la misma región no la realizan: Colombia, Bolivia, Perú y Nicaragua. En consecuencia, una imposibilidad de acceso para quienes necesiten la realización del tamizaje amerita la reflexión de hasta dónde el tamizaje de algunas enfermedades es un derecho humano (Farrell, 2008) y su estudio a la luz del principio bioético de justicia.

Así, desde diferentes teorías de justicia es posible identificar propiedades moralmente relevantes que justifican o retan la realización del tamizaje neonatal. Algunos estudios lo justifican desde una mirada utilitarista. Un análisis de 1997 en el Reino Unido demostró que el tamizaje neonatal estándar es costo-efectivo. Se cumple con los criterios de la OMS en términos de justicia distributiva que establecen que el costo de encontrar un caso (incluyendo diagnóstico y tratamiento) debe estar balanceado con los gastos posibles en cuidado médico como un todo (Kerruish y Robertson, 2005).

Otra teoría de justicia como la de las capacidades podría justificar su realización para mejorar las oportunidades de desarrollo personal e incluso social. Sin embargo, teniendo en cuenta una teoría comunitaria, debe analizarse si su realización aportaría beneficios a la sociedad. Incluso se requiere analizar en el ámbito de la normatividad local si el tamizaje neonatal corresponde a un recurso para ejercer los derechos fundamentales o un bien esencial necesario para la salud (Hernández-Álvarez, 2008), lo que justificaría su realización bajo la teoría de justicia del bienestar o la igualitaria, respectivamente.

\section{Protección normativa en tamizaje neonatal}

El tamizaje neonatal conlleva implicaciones sociales para las que se requiere un marco regulatorio. Desde sus inicios, algunos se han opuesto a su realización con el argumento de un daño social potencial por la posibilidad de eugenesia. Así es como se introdujeron protecciones procedimentales para evitar la discriminación, como la prohibición en Noruega de la recepción, posesión o uso de información del individuo derivada de una prueba genética. En otros países se establecieron estructuras regulatorias como organismos gubernamentales encargados de abordar problemas del tamizaje neonatal. En contraste, pocos países como Austria, Francia y Holanda crearon una legislación específica. Esta legislación, sin embargo, no ha sido integral, pues se ha enfocado solo en algunos aspectos como la solicitud de pruebas por un doctor especializado en genética médica y su análisis previo consentimiento escrito, o la posibilidad de rechazar un programa de tamizaje si no es científicamente confiable, el riesgo es mayor que el beneficio o entra en 
conflicto con otras normas (Chadwick et al., 1998).

Asociadas a la ausencia de un enfoque integral en la normatividad, han persistido controversias respecto a cómo debe realizarse el tamizaje neonatal. En varios estados de Estados Unidos el tamizaje es obligatorio, típicamente cuando la enfermedad por detectar tiene tratamiento, como lo sugiere la OMS. Por otro lado, en el Reino Unido, Holanda y Alemania es voluntario y se realiza cuando los padres del neonato dan su consentimiento (Raho, 2008).

Otros aspectos controversiales son la retención de muestras sobrantes del tamizaje neonatal y la limitación de los datos por obtener en aras de mantener la confidencialidad. El almacenaje de muestras se constituye en una poderosa herramienta de investigación, requiriéndose el compromiso público para evaluar los riesgos y beneficios asociados (Richer et al., 2011). En Dinamarca se cuenta con un biobanco de muestras de sangre secas de prácticamente todos los recién nacidos desde 1982, que ha sido utilizado con éxito y seguridad en varios proyectos de investigación. Este biobanco es regulado por unas guías operacionales concordantes con leyes relacionadas con el procesamiento de datos personales, derechos de los pacientes y con el comité de ética en investigación del sistema biomédico (Norgaard-Pedersen y Hougaard, 2007).
Respecto al manejo de muestras y datos hay una amplia variedad en las políticas; en Estados Unidos en algunos Estados se eliminan las muestras residuales una vez analizadas (Raho, 2008), mientras en otros conservan, y actúan incluso más allá de sus competencias legales (Lewis et al., 2011). En Alemania se eliminan luego de tres meses y además se limita el análisis a los biomarcadores a las enfermedades propuestas para tamizaje. Si la técnica identifica biomarcadores de otras enfermedades se procede a destruir estos datos. Otros países como Inglaterra y Austria conservan las muestras varios años y otros como Holanda retienen los datos colaterales para uso en caso de desarrollarse manifestaciones clínicas (Campos, 2010).

También hay controversia respecto al número de enfermedades por tamizar. Hay posiciones extremas como la de Estados Unidos donde tamizan alrededor de 54 y de Inglaterra donde tamizan cinco enfermedades. En Inglaterra para incluir un programa de tamizaje se requiere evaluar la eficacia mediante datos numéricos de obtención poco probable por la baja frecuencia y heterogeneidad de las enfermedades por evaluar (Campos, 2010). Por el contrario, en Estados Unidos los criterios para la expansión del tamizaje no están basados en evidencia, sino en recomendaciones de expertos. El ACMG recomienda el tamizaje para el mejor entendimiento de la enfermedad, el estudio sistemático de nuevas intervenciones y la oportunidad para 
incluir enfermedades en protocolos de investigación (Elliman, 2012).

En Colombia, donde solo se tamiza el hipotiroidismo congénito, la problemática es similar a la mundial. Leyes como la promulgada respecto a las enfermedades huérfanas parecen permitir la expansión del tamizaje (Bermúdez et al., 2013). No obstante, esta ley no toca aspectos fundamentales de la dimensión bioética como el consentimiento informado, las dificultades de confidencialidad o el manejo de la incertidumbre (Ley 1392, 2010). Por otra parte, el código penal colombiano no aborda el manejo de la información genética, sino solo el aspecto de la manipulación genética en su artículo 132 y el aprovechamiento ilícito de los recursos naturales en su artículo 328 , con especial referencia de la pena en que incurre quien se beneficie de los recursos genéticos de la biodiversidad colombiana (Ley 599, 2000).

Se aprecia entonces que a pesar del panorama controversial descrito hay relativamente poca cantidad de legislación dura (hard law) para abordar problemas existentes derivados del tamizaje con respecto a la gran cantidad de legislación para acercarse a inconvenientes teóricos por manipulación genética (Chadwick et al., 1998). En consecuencia, es necesario recurrir al derecho blando (soft-law), y observar que las declaraciones universales sobre genoma humano, bioética y derechos humanos e internacional sobre los datos genéticos humanos tienen anotaciones que pueden convertirse en una guía para consultar en caso de controversias en tamizaje neonatal, las cuales se analizan a continuación (ver tabla 1).

Tabla 1. Anotaciones obtenidas de declaraciones relacionadas con las consideraciones bioéticas del tamizaje neonatal.

\begin{tabular}{|c|c|}
\hline $\begin{array}{l}\text { CONSIDERACIÓN } \\
\text { BIOÉTICA }\end{array}$ & DECLARACIÓN \\
\hline Validez & $\begin{array}{l}\text { Se debe garantizar la exactitud, fiabilidad, calidad y seguridad de los datos y } \\
\text { del tratamiento de muestras biológicas, artículo } 15 \text { (Unesco, 2003). }\end{array}$ \\
\hline \multirow[t]{3}{*}{ Asesoramiento } & $\begin{array}{l}\text { Se reconoce la importancia del rigor, prudencia, probidad intelectual e integri- } \\
\text { dad en la utilización del conocimiento obtenido, artículo } 13 \text { (Unesco, 1997). }\end{array}$ \\
\hline & $\begin{array}{l}\text { Conclusiones de estudios de genética del comportamiento y poblaciones deben } \\
\text { ser cuidadosamente analizados, artículo } 7 \text { literal b (Unesco, 2003) }\end{array}$ \\
\hline & $\begin{array}{l}\text { Cuando se realicen pruebas genéticas que puedan tener consecuencias im- } \\
\text { portantes para la salud debería haber asesoramiento genético, artículo } 15 \\
\text { (Unesco, 2003). }\end{array}$ \\
\hline Riesgo-beneficio & $\begin{array}{l}\text { Un diagnóstico en relación con el genoma de un individuo solo podrá efectuarse } \\
\text { previa evaluación rigurosa de los riesgos y ventajas, artículo } 5 \text { (Unesco, 1997). }\end{array}$ \\
\hline
\end{tabular}




\title{
CONSIDERACIÓN BIOÉTICA
}

\author{
DECLARACIÓN
}

En menores de edad que no pueden expresar su aprobación, solo se podrá

Prioridad de realizar tamizajes genéticos cuando conlleven consecuencias importantes para

la persona su salud y ello responda a su interés superior, artículos 8 (Unesco, 2003) y 5e (Unesco, 1997).

Excepcionalmente se puede investigar el genoma de una persona que no obtenga un beneficio si esta redunda en beneficio para la salud de otras personas, pero a condición de no vulnerar los derechos humanos ni la ley, artículo 5e (Unesco, 1997).

Nunca pueden consentirse prácticas que sean contrarias a la dignidad humana, artículo 11 (Unesco, 1997)

Si no se está en condiciones de dar consentimiento, debe obtenerse autori-

Consentimiento zación de su representante legal, el cual debería tomar en consideración el interés superior del interesado, artículos 5 literal b (Unesco, 1997) y 8 literal b (Unesco, 2003).

Solo debería imponerse límites al consentimiento por razones poderosas del derecho interno compatible con los derechos humanos, artículo 8 literal a (Unesco, 2003).

Las muestras biológicas, así como los datos genéticos y proteómicos humanos no deberían utilizarse con fines diferentes al original o para cruzar datos a menos que se tenga un consentimiento expreso o por motivos importantes de interés público de acuerdo con el derecho interno, artículos 16a (Unesco, 2003) y 22 (Unesco, 2003).

Con personas sin la capacidad de ejercer su autonomía, se habrán de tomar medidas especiales para proteger sus derechos e intereses, artículo 5 (Unesco, 2005).

Privacidad y Los Estados deberían esforzarse por proteger la privacidad de las personas y la confidencialidad confidencialidad de los datos genéticos humanos, artículo 14a (Unesco, 2003).

Los datos genéticos y proteómicos humanos y las muestras biológicas asociadas de una persona identificable no deberían darse a conocer a terceros, salvo por razones de interés público en casos señalados en la legislación, artículo 14b (Unesco, 2003).

Los datos genéticos no deberían conservarse, de tal manera que sea posible identificar a la persona de que provienen por más tiempo del necesario para cumplir con el fin para el que fueron recolectados, artículo 14e (Unesco, 2003).

Acceso

Toda persona debe tener acceso a progresos de la medicina en materia de genoma humano, respetándose su dignidad y derechos, artículo 12 (Unesco, 1997).

Los Estados deberán respetar y promover la solidaridad con los particularmente

Solidaridad expuestos o afectados por enfermedades o discapacidades de índole genética. Se deberían fomentar investigaciones que buscan identificar, prevenir y tratar enfermedades en las que interviene la genética, artículo 17 (Unesco, 1997). 


\section{CONSIDERACIÓN}

BIOÉTICA

\section{DECLARACIÓN}

Se deberían tener en cuenta las repercusiones en generaciones futuras de las ciencias de la vida, especialmente en la constitución genética, artículo 16 (Unesco, 2005).

Cooperación internacional
Los beneficios resultantes de la utilización de datos genéticos o muestras obtenidos con fines de investigación médica y científica como acceso a la atención médica o a nuevos diagnósticos deberán ser compartidos con la sociedad y con la comunidad internacional, artículo 19 (Unesco, 2003).

Los Estados deberían regular la circulación transfronteriza de datos genéticos y muestras biológicas para fomentar la cooperación médica y científica internacional y garantizar un acceso equitativo a estos datos, y fomentar la difusión internacional de conocimientos científicos, artículo 18 (Unesco, 2003).

Fuente: elaboración propia.

Las pruebas de laboratorio por utilizar en tamizaje neonatal deben tener validez analítica, e implicar su realización en laboratorios acreditados oficialmente. La utilización de estas pruebas requiere un asesoramiento por profesionales con reconocidas calidades para solicitar, proteger, interpretar los datos obtenidos y analizar la información obtenida en el contexto del conocimiento necesario para entender su alcance, de tal manera que se obtenga una relación riesgo-beneficio favorable para el individuo y se respete la diversidad a nivel poblacional.

Desde la premisa de la prioridad de la persona por considerar al individuo un fin en sí mismo, el tamizaje neonatal debe buscar un beneficio directo para el recién nacido. Excepcionalmente podría realizarse el tamizaje neonatal sin beneficio para el recién nacido analizado siempre y cuando se beneficien otras personas y no se vulneren los derechos del neonato como ser humano. Sería moralmente cuestionable realizar un tamizaje sin beneficio para el recién nacido y que además pueda implicarle daños como estigmatización o discriminación.

Considerando que el recién nacido no está en condiciones de dar su consentimiento, el papel de la legislación es fundamental en tamizaje neonatal. El derecho interno debe definir aspectos como quién debe obrar como representante legal del neonato en situaciones especiales, como cuando los padres del menor no son los llamados a representar el interés superior del interesado, la autorización del uso de muestras y datos del tamizaje con fines diferentes al inicial por interés público, el cruce de datos y circunstancias de obligatoriedad del tamizaje en aras de proteger derechos e intereses de quienes 
carecen de capacidad para ejercer su autonomía.

Si se han realizado algunas pruebas del tamizaje neonatal con base en el interés superior del recién nacido, deben tomarse las medidas necesarias que protejan la privacidad del individuo y la confidencialidad de la información, tales como definir los datos que se obtendrán, cuando un tercero podría acceder a ellos y su tiempo de conservación, así como de las muestras de las que se obtuvieron esos datos.

Adicionalmente, se debe garantizar el acceso al tamizaje neonatal con reconocida validez y utilidad clínica, que cumplan con las consideraciones bioéticas descritas, teniendo especial atención la protección de individuos vulnerables por sus condiciones sociales. Como consecuencia de este acceso, se debe ser solidario brindando una atención integral, tanto a los enfermos como a futuras generaciones en especial riesgo de sufrir la anomalía detectada.

En este contexto, beneficios como los que conlleven mejorar los servicios de salud o reforzamiento de la investigación deben compartirse con la sociedad nacional e internacional. Para este fin, los Estados deben fomentar y regular cómo se realizaría la difusión de conocimientos científicos.

\section{Conclusiones}

Avances tecnológicos, en el diagnóstico y tratamiento de anomalías congénitas proveen oportunidades para afectar positivamente la vida de los menores afectados y sus familias (Edwards et al., 2008). Esto conlleva una gran responsabilidad dado que las personas esperan de las nuevas pruebas de tamizaje neonatal una alta exactitud y que esto evite que un niño desarrolle una enfermedad (Goldenberg et al., 2014).

Desde esta mirada, el tamizaje neonatal plantea preocupaciones en el ámbito jurídico (Dhanda y Reilly, 2003). Algunas de estas preocupaciones pueden abordarse desde el derecho blando que da importantes guías para seguir. Por ejemplo, establece la necesidad de contar con laboratorios que respalden la validez de las pruebas, la necesidad de asesoramiento profesional, en qué circunstancias se podría tamizar sin beneficio directo para el neonato o el deber de garantizar el acceso a pruebas con reconocida utilidad clínica. Sin embargo, deja situaciones para resolver por el derecho interno, con lo cual se justifica un estudio integral de la normatividad al respecto en diferentes partes del mundo y de las circunstancias especiales de esta actividad, como las relacionadas con el consentimiento y la confidencialidad. 
Por lo anterior, se justifica un debate alrededor de las consideraciones bioéticas del tamizaje neonatal. Un enfoque éticamente correcto debe aportar elementos para encontrar un equilibrio óptimo entre beneficios potenciales y posibles daños derivados de esta actividad (Orzalesi y Danhaive, 2009). Hay visiones diferentes acerca de los beneficios y los riesgos, de la importancia diferencial del impacto en salud y la sociedad, así como de la evidencia de la efectividad de una prueba. Por tanto, el abordaje de las consideraciones pertinentes requiere métodos de deliberación inclusivos y atención a los valores de los diferentes actores (Burke et al., 2010). Aspectos como evaluar la magnitud del beneficio neto en la población de tamizados para determinadas condiciones y de la capacidad para su implementación deben ser tenidos en cuenta para determinar las anomalías que se deben incluir en programas de tamizaje (Kemper et al., 2014).

En síntesis, los avances científicos hacen de los programas de tamizaje neonatal una poderosa herramienta con potencial de beneficiar no solo a los recién nacidos, sino también a toda la sociedad. No obstante, es indispensable la regulación de esta actividad con el fin de minimizar los riesgos asociados y la solución de las controversias que se presentan en su ejecución. Ante la ausencia de un abordaje legislativo integral que tenga en cuenta las consideraciones bioéticas del tamizaje neonatal, el derecho blando representa una guía para el abordaje de algunas de ellas. Sin embargo, otras consideraciones deben ser objeto del derecho interno, y ameritar así un debate bioético que considere las diversas disciplinas involucradas en esta actividad.

\section{Agradecimientos}

A la doctora Yolanda Guerra por sus comentarios

\section{Conflictos de interés}

El autor declara que no hay conflictos de interés.

\section{Referencias}

Almond, B. (2006). Genetic profiling of newborns: ethical and social issues. Nat Rev Genet, 7(1), 67-71. doi: 10.1038/ $\operatorname{nrg} 1745$.

Beauchamp, T. y Childress, J. F. (2009a). Beneficence. En Principles of Biomedical Ethics (pp. 202-248). New York: Oxford University Press.

Beauchamp, T. y Childress, J. F. (2009b). Nonmaleficence. En Principles of Biomedical Ethics (pp. 150-201). New York: Oxford University Press.

Bermúdez, A. J. , Robayo, D. B., Rosero, M. J., Valera, D. A. y Gonzalez, Y. (2013). Situational analysis to implement expanded screening in Colombia. IX Latin American Congress of Inborn Errors of Metabolism and Newborn Screening, Medellin. 
Bunnik, E. M., De Jong, A., Nijsingh, N. y De Wert, G. M. (2013). The new genetics and informed consent: differentiating choice to preserve autonomy. Bioethics, 27(6), 348-355. doi: 10.1111/ bioe. 12030 .

Burke, W., Laberge, A. M. y Press, N. (2010). Debating clinical utility. Public Health Genomics, 13(4), 215-223. doi: 10.1159/000279623.

Burke, W., Pinsky, L. E. y Press, N. A. (2001). Categorizing genetic tests to identify their ethical, legal, and social implications. American Journal of Medical Genetic, 106(3), 233-240. doi: 10.1002/ ajmg. 10011.

Campos, D. (2010). Neonatal screening by tandem mass spectrometry: an update. Revista Panamericana de Salud Publica, 27(4), 309-317. doi: S102049892010000400010 [pii].

Chadwick, R., ten Have, H., Husted, J., Levitt, M. et al. (1998). Genetic screening and ethics: European perspectives. Journal of Medicine and Philosophy, 23(3), 255-273. doi: 10.1076/ jmep.23.3.255.2580.

Chase, D. S., Tawn, E. J., Parker, L., Jonas, P. et al. (1998). The North Cumbria Community Genetics Project. Journal of Medical Genetics, 35(5), 413-416.

Colombia, Congreso de la República (2000). "Ley 599". Recuperado de http://www. alcaldiabogota.gov.co/sisjur/normas/ Normal.jsp?i=6388.

Colombia, Congreso de la República (2010). "Ley 1392". Recuperado de http://www. alcaldiabogota.gov.co/sisjur/normas/ Normal.jsp?i=39965.
De Wert, G. M. (2005). Neonatal screening: dynamics and ethics. Ned Tijdschr Geneeskd, 149(51), 2841-2843.

Dhanda, R. K. y Reilly, P. R. (2003). Legal and ethical issues of newborn screening. Pediatric Annals, 32(8), 540-546.

Edwards, E. S., Bhutani, V. K., Botkin, J., Deloian, B. et al. (2008). Newborn screening expands: Recommendations for pediatricians and medical homesImplications for the system. Pediatrics, 121(1), 192-217.

Elliman, D. (2012). Ethical aspects of the expansion of neonatal screening programme due to technological advances. Clinical Chemistry and Laboratoy Medicine, 50(6), 999-1002. doi: 10.1515/ cclm.2011.761.

Emaldi-Cirión, A. (2002). El consejo genético y la responsabilidad de los médicos que asesoran. Revista Latinoamericana de Bioética, 70-87.

Farrell, M. H., Christopher, S. A., Tluczek, A., Kennedy-Parker, K. et al. (2011). Improving communication between doctors and parents after newborn Screening. Wisconsin Medical Journal, 110(5), 221-227.

Farrell, P. M. (2008). Is newborn screening for cystic fibrosis a basic human right? Journal of Cystic Fibrosis, 7(3), 262-265. doi: 10.1016/j.jcf.2008.01.001

Goldenberg, A. J., Dodson, D. S., Davis, M. M. y Tarini, B. A. (2014). Parents' interest in whole-genome sequencing of newborns. Genetics in Medicine, 16(1), 78-84. doi: 10.1038/gim.2013.76.

Gómez, A.I. (2010). Principios éticos y jurídicos del derecho genético en las de- 
claraciones internacionales relacionadas con las intervenciones sobre el genoma humano. Vniversitas, (120), 141-168.

Gurian, E. A., Kinnamon, D. D., Henry, J. J. y Waisbren, S. E. (2006). Expanded newborn screening for biochemical disorders: the effect of a false-positive result. Pediatrics, 117(6), 1915-1921. doi: 10.1542/peds.2005-2294.

Hardart, G. E. y Chung, W. K. (2014). Genetic testing of children for diseases that have onset in adulthood: The limits of family interests. Pediatrics, 134, S104-S110.

Helgesson, G., Eriksson, S. y Swartling, U. (2007). Limited relevance of the right not to know-reflections on a screening study. Accounts of Chemical Research, 14(3), 197-209. doi: 10.1080/08989620701456322.

Hendrix, K. S., Meslin, E. M., Carroll, A. E. y Downs, S. M. (2013). Attitudes about the use of newborn dried blood spots for research: a survey of underrepresented parents. Academic Pediatrics, 13(5), 451457. doi: 10.1016/j.acap.2013.04.010.

Hernández-Álvarez, M. (2008). El concepto de equidad y el debate sobre lo justo en salud. Revista de Salud Pública, 10 $\sup (1), 72-82$.

Jonas, D.E., Wilt, T.J., Taylor, B. C., Wilkins, T. M. y Matchar, D. B. (2012). Challenges in and Principles for Conducting Systematic Reviews of Genetic Tests Used as Predictive IndicatorsMethods Guide for Medical Test Reviews (pp. 1-17). Rockville: Agency for Healthcare Research and Quality (US). Recuperado de http://www. ncbi.nlm.nih.gov/books/NBK98231/.

Kemper, A. R., Green, N. S., Calonge, N., Lam, W. K. K. et al. (2014). Decision-ma- king process for conditions nominated to the recommended uniform screening panel: Statement of the US department of health and human services secretary's advisory committee on heritable disorders in newborns and children. Genetics in Medicine, 16(2), 183-187.

Kerruish, N. J. y Robertson, S. P. (2005). Newborn screening: new developments, new dilemmas. Journal of Medical Ethics, 31(7), 393-398. doi: 10.1136/ jme.2004.008219.

Lantos, J. D. (2011). Dangerous and expensive screening and treatment for rare childhood diseases: the case of Krabbe disease. Developmental Disabilities Research Reviews, 17(1), 15-18. doi: 10.1002/ddrr.133.

Lewis, M. H., Goldenberg, A., Anderson, R., Rothwell, E. y Botkin, J. (2011). State laws regarding the retention and use of residual newborn screening blood samples. Pediatrics, 127(4), 703-712. doi: 10.1542/peds.2010-1468.

Lewis, M. H., Scheurer, M. E., Green, R. C. y McGuire, A. L. (2012). Research results: preserving newborn blood samples. Science Translational Medicine, 4(159), 159cml12. doi: 10.1126/ scitranslmed. 3004474.

Loscalzo, J., Kohane, I. y Barabasi, A. L. (2007). Human disease classification in the postgenomic era: a complex systems approach to human pathobiology. Molecular Systms Biololy, 3, 124. doi: 10.1038/msb4100163.

Massie, J. y Gillam, L. (2014). Uncertain diagnosis after newborn screening for cystic fibrosis: An ethics-based approach to a clinical dilemma. Pediatric Pul- 
monology, 49(1), 1-7. doi: 10.1002/ ppul.22933.

Matsuda, I. (2003). Bioethical considerations in neonatal screening: japanese experiences. The Southeast Asian Journal of Tropical Medicine and Public Health, 34 suppl (3), 46-48

Miller, F. A., Robert, J. S. y Hayeems, R. Z. (2009). Questioning the consensus: managing carrier status results generated by newborn screening. American Journal of Public Health, 99(2), 210-215. doi: 10.2105/ajph.2008.136614.

Norgaard-Pedersen, B. y Hougaard, D. M. (2007). Storage policies and use of the Danish Newborn Screening Biobank. Journal of Inherited Metabolic Disease 30(4), 530-536. doi: 10.1007/s10545007-0631-x.

Ortiz, T. (2008). Programa Nacional de Tamizaje y Diagnóstico Genético. Curso Continuo de Actualización en Pediatría, 7, 14-18. Recuperado de https://scp. com.co/precop/precop_files/modulo_7_vin_2/14-18\%20Programa\%20 tamizaje.pdf.

Orzalesi, M. y Danhaive, O. (2009). Ethical problems with neonatal screening. Annali dell'Istituto Superiore di Sanita, 45(3), 325-330.

President's Council on Bioethics (2008). The changing moral focus of newborn screening, an ethical analysis. Recuperado de https://repository.library.georgetown.edu/ bitstream/handle/10822/559367/Newborn\%20Screening\%20for\%20the\%20 web.pdf? sequence $=1$ \& risAllowed $=y$.

Raffan, E. y Semple, R. K. (2011). Next generation sequencing -implications for clinical practice. British Medical Bulletin, 99, 53-71. doi: 10.1093/bmb/ldr029.

Raho, J.A. (2008). The changing moral focus of newborn screening, an ethical analysis by the President's Council on Bioethics. Appendix newborn screening: an international survey. Recuperado de https://repository.library.georgetown.edu/bitstream/ handle/10822/559379/the\%20changing $\% 20$ moral\%20focus $\% 20$ of\%20 newborn $\% 20$ screening $\% 20-\% 20$ appendix\%20-\%20joseph\%20raho. pdf? sequence $=1$ \&risAllowed $=y$.

Richer, J., Ghebremichael, M. S., Chudley, A. E., Robinson, W. M., W. et al. (2011). Research use of leftover newborn bloodspots: attitudes of Canadian geneticists regarding storage and informed consent requirements. Genetics in Medicine, 13(4), 305-313. doi: 10.1097/GIM.0b013e3181f69da0.

Rose, N. C. y Dolan, S. M. (2012). Newborn screening and the obstetrician. Obstetrics \& Gynecology, 120(4), 908917. doi: 10.1097/AOG. Ob013e31826b2f0300006250- 20121000000023 [pii].

Sánchez, C. (2002). El derecho a la protección de la intimidad de los datos genéticos. Revista Latinoamericana de Bioética, 104-115.

Schiffman, J. D., Geller, J. I., Mundt, E., Means, A. et al. (2013). Update on pediatric cancer predisposition syndromes. Pediatric Blood E Cancer, 60(8), 1247-1252. doi: 10.1002/pbc.24555.

Schulman, A. (2008). The Future of newborn screening: clouds on the horizon? Recuperado de https://bioethicsarchive. 
georgetown.edu/pcbe/background/newborn_screening_schulman.html.

Strachan, T. y Read, A. (2011). Genes in pedigrees and populations. En Human Molecular Genetics (4ta. edición) (pp. 61-90). New York: Garland Science, Taylor \& Francis Group.

Tarini, B. A. (2007). The current revolution in newborn screening: new technology, old controversies. The Archives of Pediatrics $\&$ Adolescent Medicine, 161(8), 767 772. doi: 10.1001/archpedi.161.8.767.

Unesco (1997). Declaración Universal sobre el Genoma Humano y los Derechos Humanos. Recuperado de http://portal.unesco.org/
es/ev.php-URL_ID=13177\&URL_DO=DO_TOPIC\&URL_SECTION=201. html.

Unesco (2003). Declaración Internacional sobre los Datos Genéticos Humanos.Recuperado. de http://portal.unesco.org/ es/ev.php-URL_ID=17720\&URL_DO=DO_TOPIC\&URL_SECTION=201. html.

Unesco (2005). Declaración Universal sobre Bioéticay Derechos Humanos. Recuperado de http://portal.unesco.org/es/ev.phpURL_ID=31058\&URL_DO=DO_TOPIC\&URL_SECTION=201.html. 